\title{
CYGNUS DIVERTER SWITCH ANALYSIS *
}

\author{
E. Ormond ${ }^{\xi}$, I. Molina, S. Cordova, D. Nelson \\ Sandia National Laboratories, PO Box 238, Mail Stop 944 \\ Mercury, NV 89023 USA
}

\author{
J. Smith \\ Los Alamos National Laboratory, PO Box 1663, Mail Stop D410 \\ Los Alamos, NM 87545 USA
}

\author{
G. Corrow, M. Hansen, D. Henderson, C. Mitton \\ National Securities Technologies, 2621 Losee Rd \\ North Las Vegas, NV 89030 USA
}

\begin{abstract}
The Cygnus Dual Beam Radiographic Facility consists of two 2.25-MV, 60-kA, 50-ns x-ray sources fielded in an underground laboratory at the Nevada Test Site. The tests performed in this laboratory involve study of the dynamic properties of plutonium and are called subcritical experiments. From end-to-end, the Cygnus machines utilize the following components: Marx generator, water-filled pulse-forming line (PFL), waterfilled coaxial transmission line (WTL), 3-cell inductive voltage adder (IVA), and rod-pinch diode. The upstream WTL interface to the PFL is via a radial insulator with coaxial geometry. The downstream WTL terminates in a manifold where the center conductor splits into three lines which individually connect to each of the IVA cell inputs. There is an impedance mismatch at this juncture. It is a concern that a reflected pulse due to anomalous behavior in the IVA or diode might initiate breakdown upon arrival at the upstream PFL/WTL insulator. Therefore near the beginning of the WTL a radial diverter switch is installed to protect the insulator from over voltage and breakdown. The diverter has adjustable gap spacing, and an in-line aqueous-solution (sodium thiosulfate) resistor array for energy dissipation. There are capacitive voltage probes at both ends of the WTL and on the diverter switch. These voltage signals will be analyzed to determine diverter performance. Using this analysis the usefulness of the diverter switch will be evaluated.
\end{abstract}

I. GENERAL SYSTEM DESCRIPTION

The Cygnus Dual Beam Radiographic Facility consists of two Flash X-Ray machines designed for the Armando Subcritical Experiment (SCE) at the Nevada Test Site (NTS). Their success led to a series of experiments named Thermos which concluded in May of 2007.

The machines had to fit in an underground laboratory and as such were designed in a linear fashion. The other requirement was two machines that could be fired independently and 60 degrees apart as shown in Figure 1. The major components of Cygnus include the Marx, PFL, WTL, IVA, Vacuum Insulated Transmission Line (VITL) and Diode.

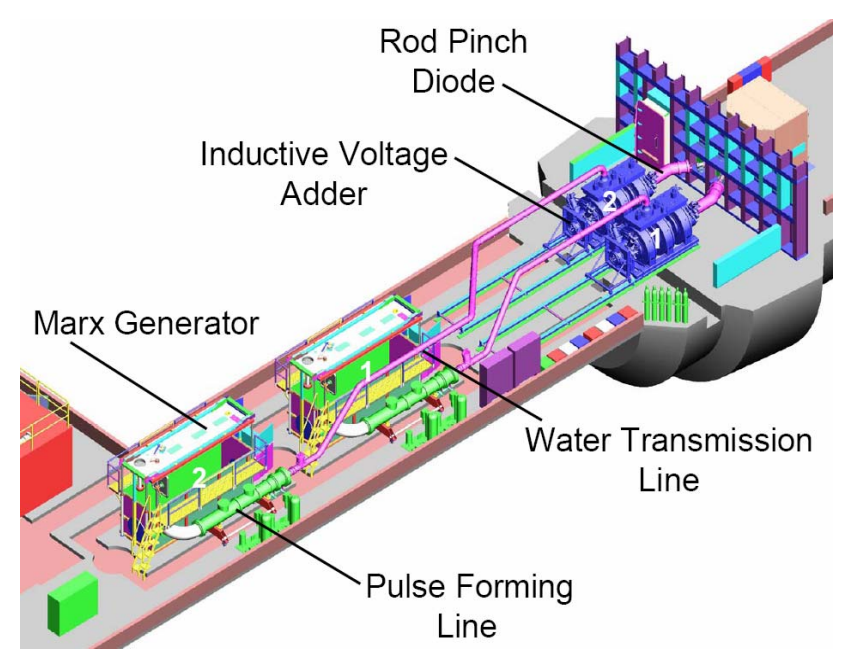

Figure 1. Cygnus layout in the U1a facility at the Nevada Test Site.

\footnotetext{
* Sandia is a multi-program laboratory operated by Sandia Corporation, a Lockheed Martin Company, for the United States Department of Energy's National Nuclear Security Administration under contract DE-AC04-94AL85000.

گ email: eormond@ sandia.gov
} 


\section{A. Marx}

The Marx consists of 32 full stages of $100 \mathrm{kV}, 200 \mathrm{nF}$ capacitors and two half-stages of $50 \mathrm{kV}$ capacitors. The Marx output is directed with a swing arm to either a 28 ohm load or to the PFL. The swing arm allows the Marx to be charged while the output is tied to the dummy load. If a pre-fire occurs during charging it is safely absorbed in the dummy load while protecting the diode. This is a valuable feature as the diode requires rebuilding after every down-line pulse.

The Marx is suspended on nylon straps from the Marx tank lid as shown in Figure 2. This allows maintenance by simply raising the Marx lid out of the oil using hydraulic rams located on each corner of the Marx tank.

The Marx diagnostics include a Current Viewing resistor (CVR) on the input of the Marx and a voltage monitor attached to the output plate.

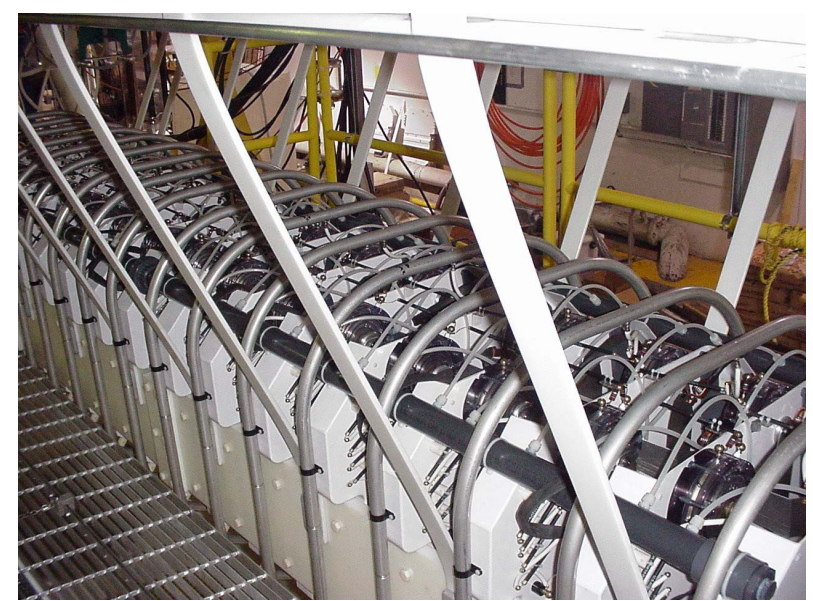

Figure 2. Cygnus Marx Generator on nylon straps.

\section{B. PFL}

The PFL is a modified Radiographic Integrated Test Stand (RITS) design [1] that was shortened approximately six inches. The main switch, transfer lines, and sharpening switch are the original RITS design. However the oil pre-pulse switch was modified to allow transition to the WTL (Fig 3).

The diagnostics on the PFL include current (B-dot) and voltage (D-dot) monitors located before and after the PFL and sharpening switches.

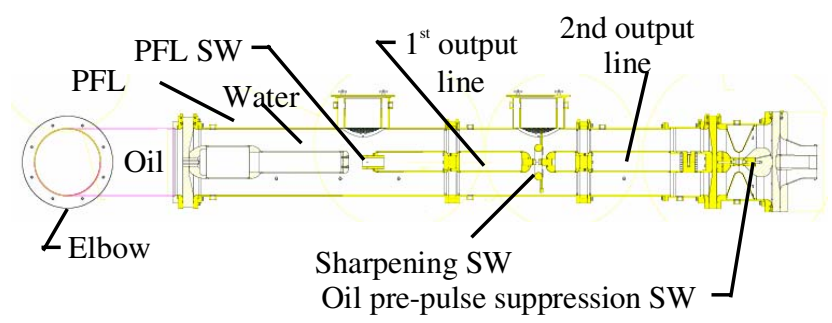

Figure 3. Cygnus PFL.

\section{WTL and Diverter Switch}

The PFL output transitions to a WTL which allows flexibility in placement of the Marx/PFL in the underground facility. Water conditioning stations keep the water de-ionized and de-aerated while maintaining conductivity greater than $10 \mathrm{M}$ per square centimeter. Diagnostics include B-dot and D-dot monitors on the input and output of the WTL.

The Diverter Switch uses five sodium thiosulfate water resistors to absorb reflected energy and protect the PFL output barrier. A torlon rod can be adjusted to vary the gap as shown in Figure 4.

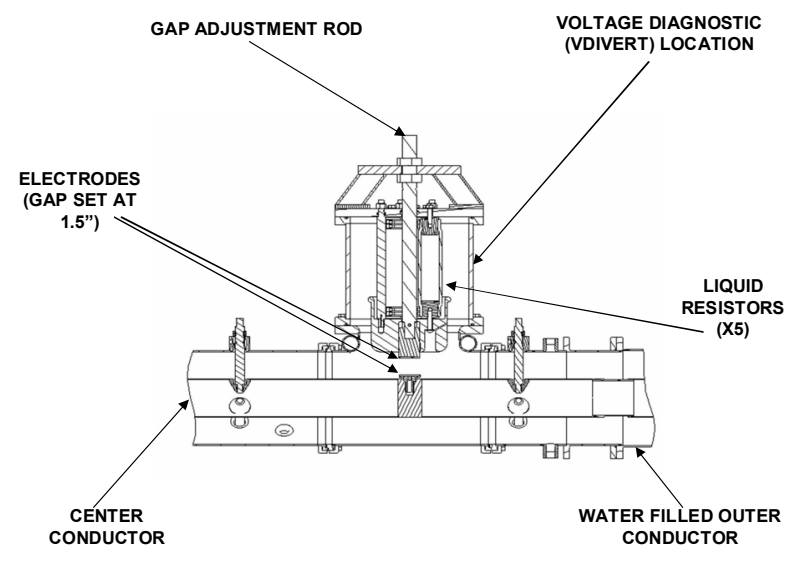

Figure 4. Cygnus Diverter Switch cutaway.

An additional D-dot was added approximately mid-point in line with one of the five resistors to measure the reflected pulse as shown in Figure 4.

\section{IVA and VITL}

The IVA skid includes an oil manifold and three IVA cells feeding a VITL. The cells are modified Sandia Accelerator and Beam Research Experiment (SABRE) cells which have a new radial vacuum feed. The cells also have access plates for easy inspection and cleaning. All surfaces inside the cells and VITL exposed to greater than $150 \mathrm{kV} / \mathrm{cm}$ have been hard anodized to control surface emission.

A cantilevered stalk is adjusted using four vacuum bellows attached to cables. A rotational adjustment was added to allow alignment of the 30 degree bend in the VITL towards the diode.

\section{E. Diode}

Cygnus uses the Rod Pinch diode developed by the Naval Research Laboratory [2] and tested on TRIMEV [3] and SABRE.

\section{HISTORY OF THE DIVERTER SWITCH}

The need for a Diverter Switch to absorb reflected energy was addressed during the design stage for the Cygnus machines [4]. Weidenheimer theorized that for 
anomalous conditions the reflected energy from a reverse polarity spike, $10 \mathrm{~ns}$ in duration, could be $80 \%$ of the forward going pulse amplitude. Computer modeling predicted the most stressed node would be the WTL to PFL transition. This barrier is a 2" thick barrier manufactured from polycarbonate.

The Diverter switch was added due to this possible reflected pulse that could potentially damage the output barrier of the PFL.

\section{ANALYSIS OF THE DIVERTER SWITCH}

Review of the diverter switch waveforms has proven to be instrumental in determining degradation of torlon rods used in the WTL. It also gave an indication when tracking of the PFL input barrier occurred.
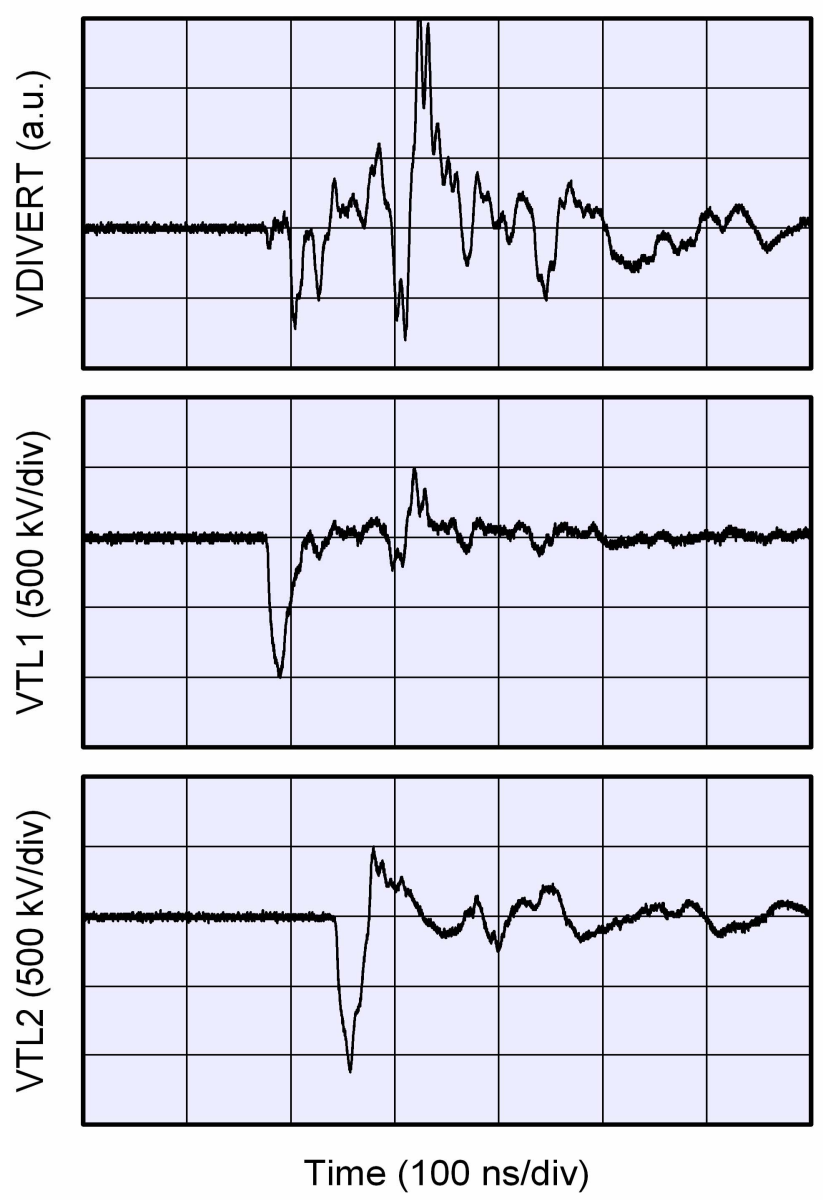

Figure 5. Cygnus 1 VDIVERT, VTL1 and VTL2.

The wave shape was analyzed to see how it correlates to diode operation and failure modes. Standard comparisons of the VDIVERT (Fig 4), VTL1 (near the beginning of the WTL) and VTL2 (near the end of the WTL) for each machine are shown in Figure 5 for Cygnus 1 and Figure 6 for Cygnus 2.
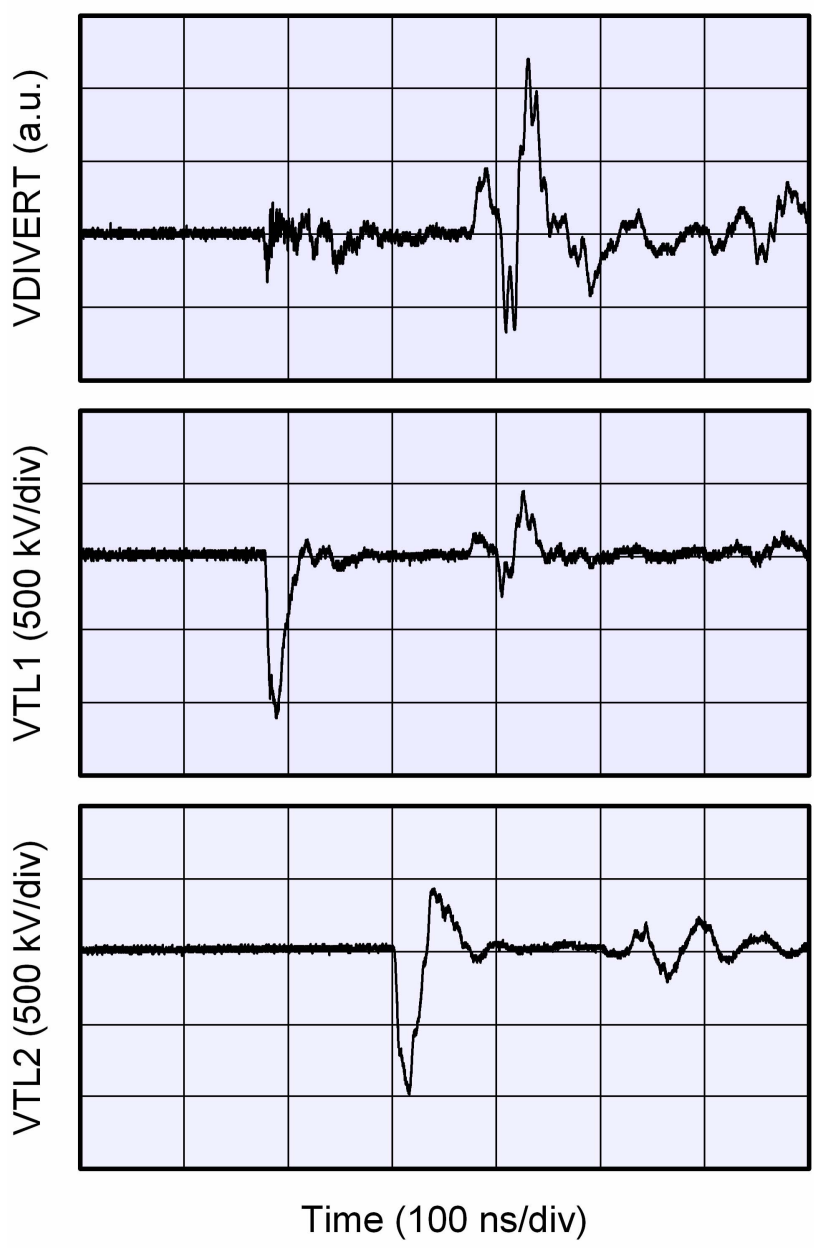

Figure 6. Cygnus 2 VDIVERT, VTL1 and VTL2.

\section{A. Basic Diverter Wave Shape}

On the VDIVERT trace the down-line pulse is labeled as "Main Pulse" which is shown in Figures 7, 8, and 9. The "Reflected Pulse" (inverted) is the positive pulse being adsorbed by the switch. The signal between the main pulse and the reflected pulse was defined as prepulse and was further divided in to "No pre-pulse", "Small pre-pulse" and "Large pre-pulse" which is shown in Figures 7, 8, and 9. These three wave shapes indicate increasing degrees of pre-pulse. The increasing pre-pulse was not an indicator of breakdowns.

- $\quad$ No pre-pulse is identified in Figure 7 and shows a noisy baseline until the reflected pulse returns.

- $\quad$ Small pre-pulse shows a small pulse just before the reflected pulse in Figure 8.

- Large pre-pulse shows multiple transitions as shown in Figure 9. 


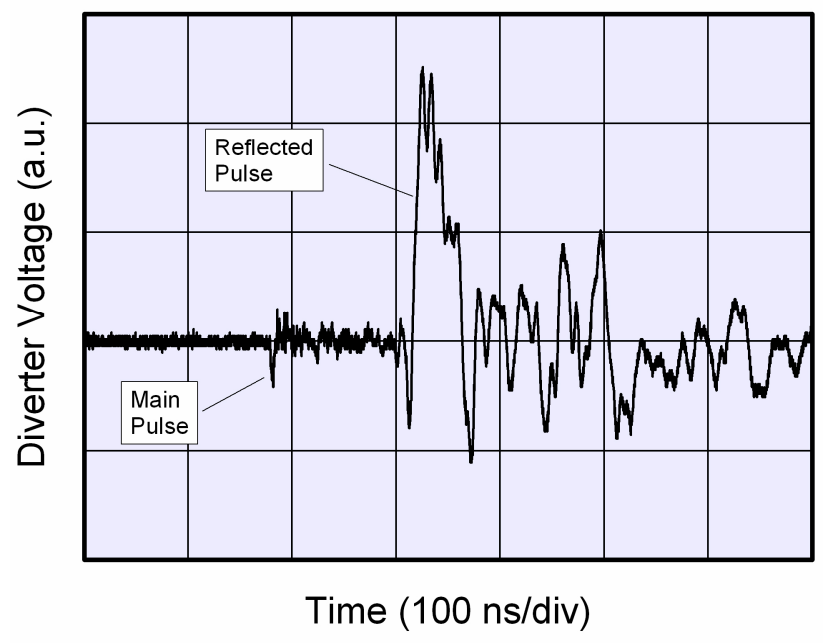

Figure 7. Sample of No Pre-pulse wave form.

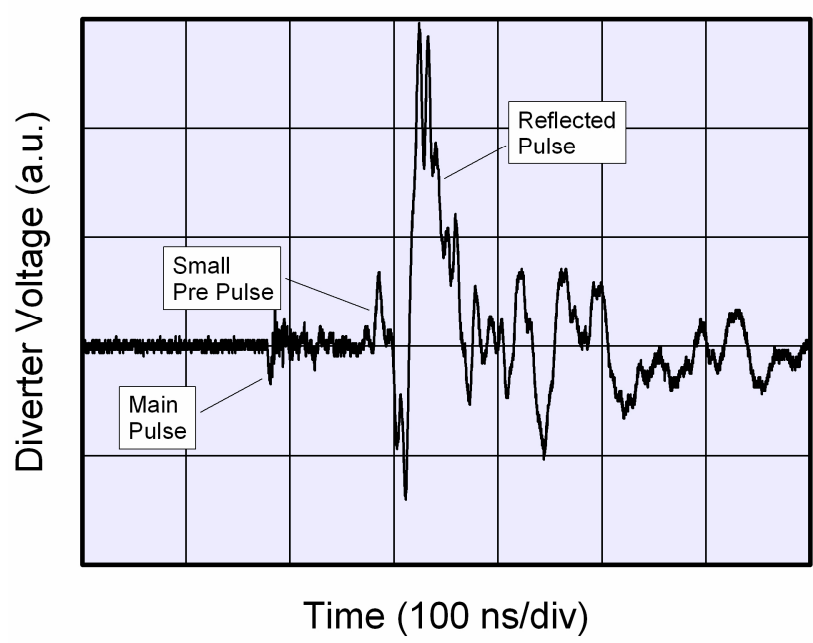

Figure 8. Sample of Small Pre-pulse wave form.

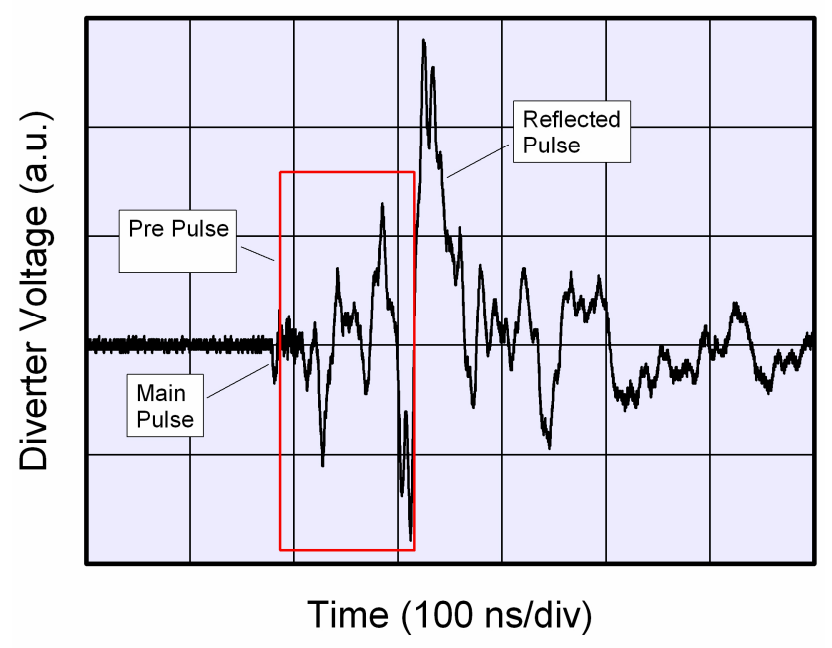

Figure 9. Sample of Large Pre-pulse wave form.

The last shape, "Large Pre-pulse" was observed often. Comparisons of the WTL input diagnostic (VTL1) to the output diagnostic (VTL2) were completed to verify this absorbed energy did not limit power to the IVA manifold.

\section{B. Integral}

The switch energy absorbed was important since the original intent of the analysis was to determine if the switch was needed at all. The resistor stack was designed to absorb the entire outgoing pulse if the gap was incorrectly set so the safety of the PFL output barrier is the only part in question.

The original calibration data is limited therefore it is not possible to definitively state if the diverter switch is required in the system. Further calibrations will be performed to allow this determination.

Though the energy measured has arbitrary units, the data will be ready to manipulate once the calibration factor is determined. Table 1 shows all down line shots since the machines were moved under ground in 2003. Despite occasional torlon rod failures in the WTL, the VDIVERT integral has been very consistent. Note that during the Armando series on Cygnus 2 the VDIVERT integral was $730+/-44$. During this series of shots there were no faults anywhere in the machine.

Table 1. Cygnus Diverter energy.

\begin{tabular}{|l|c|c|}
\hline & $\begin{array}{c}\text { Cygnus 1 } \\
\text { (a.u.) }\end{array}$ & $\begin{array}{c}\text { Cygnus 2 } \\
\text { (a.u.) }\end{array}$ \\
\hline $\begin{array}{l}\text { Armando } \\
\text { Confirmatory }\end{array}$ & $912 \pm 103$ & $783 \pm 58$ \\
\hline Armando & $850 \pm 99$ & $730 \pm 44$ \\
\hline Step Wedge & $864 \pm 94$ & $779 \pm 62$ \\
\hline $\begin{array}{l}\text { Thermos } \\
\text { Confirmatory }\end{array}$ & $993 \pm 55$ & $671 \pm 60$ \\
\hline Thermos & $1055 \pm 54$ & $763 \pm 46$ \\
\hline All Shots & $963 \pm 109$ & $740 \pm 70$ \\
\hline
\end{tabular}

\section{DIAGNOSTIC IMPORTANCE}

Review of the diverter switch data has proven the VDIVERT diagnostic voltage monitor to be crucial in determining degradation of the torlon rods used in the WTL and indicated tracking of the main MARX/PFL barrier.

Cygnus reliability has always been a key operational element. The risk inherent in the execution of a SCE is that a high-stakes package is expended in a single event where there is no reprieve from catastrophic equipment failure [5].

When the Cygnus machines are operating flawlessly the diagnostic produces a very repeatable waveform that gives the operators a quick look at the machine health as shown in the ten shot overlay of Figure 10. 


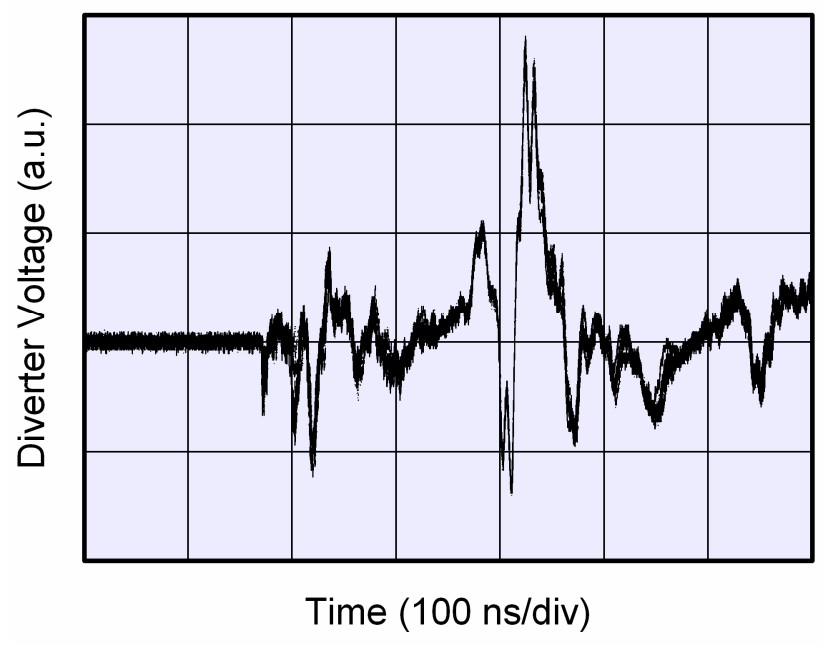

Figure 10. Standard 10 Shot Overlay.

The benefit of the diverter switch as a diagnostic is shown in Figure 11 as we compare a standard shot (blue waveform) to a torlon rod failure (red waveform). There is no reflected pulse for a torlon rod failure.

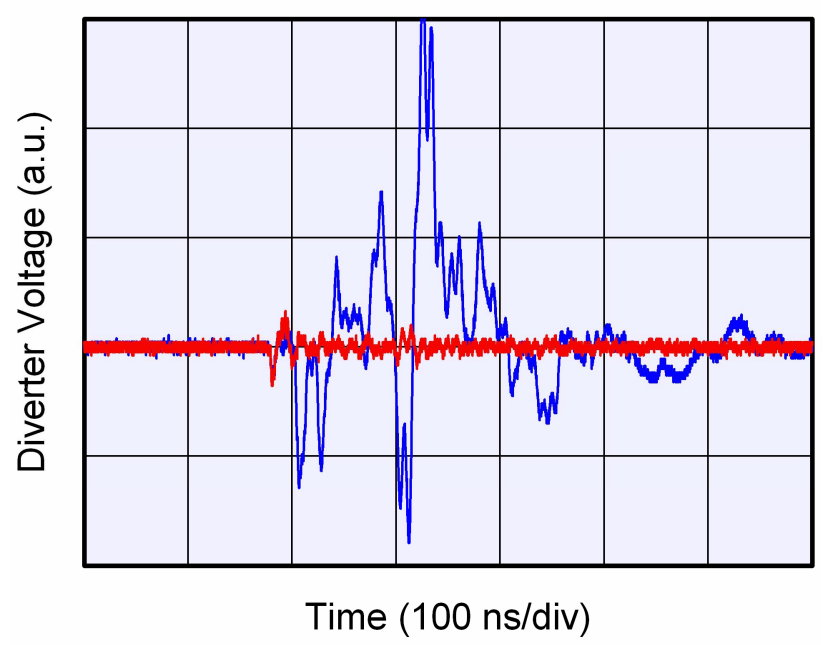

Figure 11. Anomaly Comparison where blue is a standard shot and red is a torlon rod failure.

\section{SUMMARY}

The diverter switch has been shown to be a valuable diagnostic. Even if future calibration and analysis proves it is not required as a protective feature we would be inclined to design a diagnostic to remain in the same location for future analysis of the torlon rod issues and PFL barrier studies.

The existence of pre-pulse did not have any noticeable affect on the output of the machines. It was theorized that power absorbed during the outgoing pulse would have a direct effect on dose, but review of dose level to pre-pulse activity showed no correlation.
The diverter diagnostic has become a critical diagnostic during post shot analysis as it has a high sensitivity to failures starting at the PFL and continuing up to an impedance collapse in the diode.

\section{ACKNOWLEDGMENTS}

The authors gratefully acknowledge the drafting skills of Deanna Jaramillo of Sandia National Laboratories.

\section{REFERENCES}

[1] D. Johnson et al., "Magnetic Insulation, Power Flow, and Pulsed Power Results on RITS-3," CP650, BEAMS 2002, Proc. $14^{\text {th }}$ International Conference on High Power Particle Beams, pp. 123-126.

[2] G. Cooperstein et al., "Theoretical Modeling and experimental Characterization of a Rod-Pinch Diode," in Physics of Plasmas, Vol. 8, Number 10, October 2001.

[3] P. Menge et al., SAND Report SAND2002-0082, "Experimental Comparison of 2-3MV X-ray Sources for Flash Radiography" Sandia National Laboratories, January 2002.

[4] D. Weidenheimer et al., "Design of a driver for the Cygnus x-ray source" in Proceedings of the $13^{\text {th }}$ IEEE Pulsed Power Conf., 17-22 June 2002, pp. 591-595.

[5] J. Smith et al., "Performance of the Cygnus x-ray source" in Proceedings of the $15^{\text {th }}$ IEEE Pulsed Power Conf., 13-17 June 2005, pp. 334-337.

This manuscript has been authored by National Security Technologies, LLC, under Contract No. DE-AC52-06NA25946 with the U.S. Department of Energy. The United States Government retains and the publisher, by accepting the article for publication, acknowledges that the United States Government retains a non-exclusive, paid-up, irrevocable, world-wide license to publish or reproduce the published form of this manuscript, or allow others to do so, for United States Government purposes. 